\title{
Numerical Analysis of Indirect Combustion Noise Generation Within a Subsonic Nozzle
}

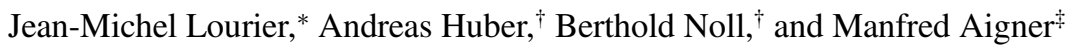 \\ DLR, German Aerospace Center, 70597 Stuttgart, Germany
}

DOI: $\underline{10.2514 / 1 . J 052755}$

\begin{abstract}
Indirect noise is analyzed by numerical simulations of the Entropy Wave Generator experiment, which has been set up to study the fundamental mechanism of indirect combustion noise generation. Previous studies of this test case show the major importance of modeling the impedance terminations of the test rig to obtain accurate results. So far, characteristic boundary conditions have been used to model the impedance for numerical calculations. Within this paper, different proposals for setting up the characteristic boundary conditions are reviewed. In addition, a higher accuracy of the impedance model is achieved by applying time-domain impedance boundary conditions. As part of the Entropy Wave Generator experiment, indirect noise is generated by electrical heating wires. It has been found in this study that the modeling of these heat sources is crucial for obtaining accurate results. An advanced heat source model is proposed that is in excellent agreement with the experimental data. Both the advanced impedance and heat source model account for a better agreement between experimental and numerical data than reported in literature to date.
\end{abstract}

\section{Nomenclature}

$a, b=$ impedance model constants

$c \quad=$ speed of sound, $\mathrm{m} / \mathrm{s}$

$D_{\Phi} \quad=$ dissipation associated terms

$F_{s} \quad=$ downsampling factor

$f \quad=$ frequency, $\mathrm{Hz}$

$H=$ acoustic transfer function

$h \quad=$ sensible enthalpy, $\mathrm{W}$

$j \quad=$ imaginary unit

$K=$ relaxation coefficient, $1 / \mathrm{s}$

$L=$ characteristic wave, $\mathrm{Pa} / \mathrm{s}$

$l \quad=$ length, $\mathrm{m}$

$M a=$ Mach number

$p \quad=$ pressure, $\mathrm{Pa}$

$R=$ reflection factor

$R_{\Phi} \quad=$ pressure associated terms

$s=$ propagation speed, $\mathrm{m} / \mathrm{s}$

$T \quad=$ temperature, $\mathrm{K}$

$t=$ time, $\mathrm{s}$

$t_{D} \quad=\quad$ delay time, $\mathrm{s}$

$t_{P} \quad=\quad$ heating pulse duration, $\mathrm{s}$

$t_{0} \quad=$ starting time, $\mathrm{s}$

$u=$ velocity, $\mathrm{m} / \mathrm{s}$

$w_{k} \quad=$ state variable, $\mathrm{m} / \mathrm{s}$

$X, Y=$ input and output in $z$ space

$x, y=$ input and output in time-space

$x_{h} \quad=\quad$ location of heating wire, $\mathrm{m}$

$x_{i}=$ spatial coordinate, $\mathrm{m}$

$Z=$ impedance, $\mathrm{kg} / \mathrm{m}^{2} / \mathrm{s}$

$\alpha^{+}, \alpha^{-}=$wave amplitudes, $\mathrm{Pa}$

$\Delta t=$ time step, $\mathrm{s}$

$\Delta x=$ mesh spacing, $\mathrm{m}$

Presented as Paper 2013-1142 at the 51st Aerospace Sciences Meeting, Grapevine, TX, 7-10 January 2013; received 15 May 2013; revision received 27 September 2013; accepted for publication 13 October 2013; published online 26 March 2014. Copyright (C) 2013 by German Aerospace Center (DLR). Published by the American Institute of Aeronautics and Astronautics, Inc., with permission. Copies of this paper may be made for personal or internal use, on condition that the copier pay the $\$ 10.00$ per-copy fee to the Copyright Clearance Center, Inc., 222 Rosewood Drive, Danvers, MA 01923; include the code $1533-385 \mathrm{X} / 14$ and $\$ 10.00$ in correspondence with the CCC.

*Ph.D. Student, Institute of Combustion Technology, Pfaffenwaldring 38-40. Student Member AIAA.

Research Engineer, Institute of Combustion Technology, Pfaffenwaldring 38-40.

+Director, Institute of Combustion Technology, Pfaffenwaldring 38-40.

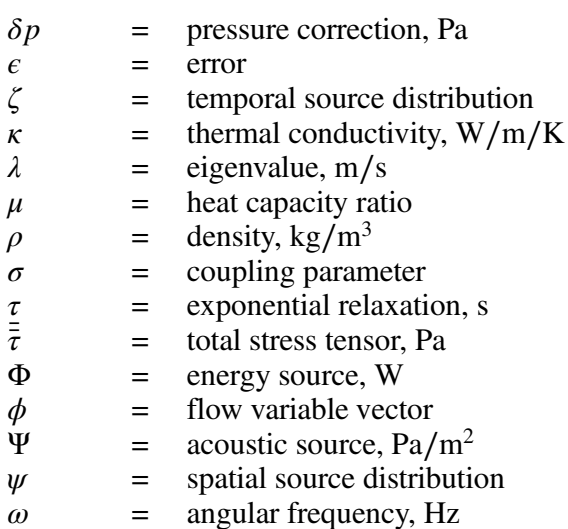

Subscripts

$\begin{array}{lll}i & = & \text { spatial direction } \\ k & = & \text { summation or multiplication index } \\ m & = & \text { characteristic mode } \\ s & = & \text { specified value } \\ \infty & = & \text { far-field conditions }\end{array}$

Superscripts

$n, *, \delta=$ time levels

\section{Introduction}

A IRCRAFT engine noise has been reduced drastically over the last few decades [1]. Especially the main noise sources of jet engines (i.e., fan and jet noise [2]) have been attenuated, leading to a growing importance of minor noise sources such as combustion noise [1]. Combustion noise can be divided into direct and indirect combustion noise. In case of the former, sound waves are generated by unsteady heat release rates in the combustion chamber $[3,4]$. In contrast, indirect combustion noise is generated by accelerated entropy modes [5-7]. Bake et al. [7] set up a generic test case for indirect combustion noise, namely the Entropy Wave Generator (EWG) test case. The EWG consists of a converging-diverging nozzle flow where entropy modes are generated upstream of the nozzle by electrical heating wires. These entropy modes are accelerated through the nozzle, which leads to pressure fluctuations (i.e., indirect noise).

The EWG test case has been investigated with experimental $[7,8]$, analytical [5,9-11], and numerical methods [7,9,10,12]. From the analytical and numerical studies, it has been found that the modeling 
of the downstream impedance of the test rig is of major importance to obtain accurate results. Mühlbauer et al. [12] and Leyko et al. [9] have shown that this impedance can be modeled numerically with a first-order filter, which is applied by Navier-Stokes characteristic boundary conditions (NSCBCs) $[13,14]$. Thus, the impedance is tuned by means of a coupling parameter to fit the experimental results. Even though Mühlbauer et al. [12] and Leyko et al. [9] use the same boundary conditions, they propose very different values for the NSCBC coupling parameter. In this paper, the different proposals for modeling the downstream impedance with NSCBC are compared by means of analytical and numerical data. Previous reports $[9,10,12]$ propose to apply the experimentally measured impedance by Bake et al. [7] as further advancement of the numerical calculations. This is put into practice in this work by applying time-domain impedance boundary condition (TDIBC) in the numerical simulations of the EWG test case $[15,16]$.

Besides the impedance termination of the EWG test rig, the modeling of the heat sources has a major impact on the accuracy of the results as shown in the current study. However, very different modeling approaches for the spatial and temporal distribution of the sources are reported in literature $[9,10,12]$. These approaches are analyzed in this work, and an improved model for the heat sources of the subsonic EWG reference test case is proposed.

For this discussion, the subsonic EWG reference test case is analyzed by means of the computational fluid dynamics (CFD) solver DLR THETA $[17,18]$. The solution algorithm and the boundary conditions of the applied solver are discussed in Secs. II and III, respectively. Both the experimental and the numerical setup of the EWG test case are shown in Sec. IV. Finally, results are presented in Sec. Vand discussed with respect to the different modeling approaches.

\section{Fractional Step Method}

The Navier-Stokes equations for compressible flows are solved by means of a fractional step method $[19,20]$ proposed by Moureau et al. [21], which is henceforth called the semi-implicit characteristic splitting (SICS) solver. Generally speaking, fractional step methods split a physical time step into multiple computational steps (e.g., into a predictor and a corrector step). The operators of the underlying equations are decomposed and solved separately within the different computational steps [19]. In case of SICS, the Navier-Stokes equations are decomposed by means of a characteristic splitting into acoustic and advective modes.

The Navier-Stokes equations for compressible flows can be arranged as [21]

$$
\begin{gathered}
\frac{\partial \rho}{\partial t}+\rho \nabla \cdot u+\underline{u \cdot \nabla \rho}=0 \\
\frac{\partial \rho u}{\partial t}+\rho u \nabla \cdot u+\underline{u \cdot \nabla \rho u}=\underline{-\nabla p}+\nabla \cdot \overline{\bar{\tau}} \\
\frac{\partial \rho h}{\partial t}+\rho h \nabla \cdot u+\underline{u \cdot \nabla \rho h}=\underline{-c^{2} \rho \nabla \cdot u}+\tau \nabla \cdot(\kappa \nabla T)+\gamma \overline{\bar{\tau}} \cdot \nabla u
\end{gathered}
$$

As shown by Moureau et al. [21], the previous systems of equations can be split into one system with only advective and another with only acoustic modes. In this scope, the eigenvalues of Eqs. (1-3) are computed. To be able to compute the eigenvalues, the diffusive terms are neglected. Furthermore, the flow is assumed to be onedimensional for simplicity. Under these assumptions, the eigenvalues of Eqs. (1-3) amount to $[u-c, u, u+c]$. The first and last eigenvalues are equal to the speed at which acoustic waves propagate through a one-dimensional flow. Hence, the initial system of equations includes, among others, acoustic modes. As shown by Moureau et al. [21], the speed of sound $c$ can be removed from these eigenvalues by neglecting the underlined terms in Eqs. (1-3), which is equal to removing the acoustic modes from the system of equations. Neglecting the underline terms results in the following system of equations:

$$
\frac{\phi^{*}-\phi^{n}}{\Delta t}+u \cdot \nabla \phi=D_{\phi}
$$

where $\phi$ is $[\rho, \rho u, \rho h]$, and $D_{\phi}$ is $[0, \nabla \cdot \mathbf{t}, \gamma \nabla \cdot(\kappa \nabla T)+\gamma \overline{\bar{\tau}} \cdot \nabla u] . \phi^{*}$ is an intermediate solution. Computing the eigenvalues of Eq. (4), with the same assumptions used before, results in $[u, u, u][2 \overline{1}]$. Therefore, Eq. (4) is an advection equation that propagates the values of $\phi$ at the flow speed $u$. In comparison to the eigenvalues of the initial system of Eqs. (1-3), the speed of sound is removed from the first and last eigenvalues (i.e., the acoustic modes are removed from the initial equations).

Besides the advective system of equations, a system with pure acoustic modes is obtained by subtracting Eq. (4) from Eqs. (1- $\underline{-3})$ :

$$
\frac{\phi^{n+1}-\phi^{*}}{\Delta t}+\nabla \phi \cdot u=R_{\phi}
$$

where $R_{\phi}$ is $\left[0,-\nabla p,-c^{2} \rho \nabla \cdot u\right]$. The eigenvalues of Eq. (5) amount to $[-c, 0, c][21]$. All disturbances propagate at the speed of sound, and hence the previous equation includes only the acoustic modes.

As mentioned previously, the advective and acoustic systems are used as predictor and corrector steps, respectively, of the fractional step method SICS. Within the predictor step, Eq. (4) is solved subsequently in a semi-implicit way. By contrast, the acoustic system is solved implicitly to remove the acoustic CFL limitation [21]. For this reason, Eq. (5) is transposed to form a wave equation:

$$
\begin{gathered}
\nabla \cdot \nabla \delta p-\nabla \cdot \frac{2 u}{c^{2} \Delta t} \delta p-\frac{4}{c^{2} \Delta t^{2}} \delta p=-\nabla \cdot \nabla\left(p^{n}+p^{*}\right) \\
+\frac{4}{\Delta t}\left(\frac{\rho^{*}-\rho^{n}}{\Delta t}+\nabla \cdot \frac{\rho u^{*}+\rho u^{n}}{2}\right)
\end{gathered}
$$

where the pressure correction $\delta p$ reads

$$
\delta p=p^{n+1}-p^{*}
$$

As part of the derivation of Eq. (6), the continuity of Eq. (1) is used. Any defect in mass conservation of the intermediate solution $\phi^{*}$ gives rise to a source contribution on the RHS of the wave Eq. (6). This ensures that the final solution $\phi^{n+1}$ is mass conservative, i.e., the continuity Eq. (1) is satisfied. In addition, this also guarantees that the acoustic and convective modes are coupled properly in the final solution. For the final solution, the results of the two fractional steps are superimposed, which allows both acoustic and convective modes to propagated within the solution. More details of the solution algorithm are shown in [21].

\section{Acoustic Boundary Conditions}

The computational domain of a CFD simulation usually only comprises a part of the physical domain due to limitations of computational resources and turnaround times [22]. Therefore, boundary models have to be introduced. In the case of hyperbolic systems, such as the wave equation discussed here, artificial boundaries are usually derived from the analysis of different waves crossing the boundary [23]. The classical approach for this is the Navier-Stokes characteristics boundary conditions [13]. Generally speaking, characteristic equations are solved at boundaries, allowing for a separate treatment of boundary crossing waves.

\section{A. Navier-Stokes Characteristic Boundary Conditions}

To derive the NSCBCs, the underlying equations have to be written in characteristic form $[13,23]$. A characteristic analysis of the Navier-Stokes Eqs. (1-3) shows that five characteristic waves $L_{m}$ cross inlet and outlet boundaries as shown in Fig. 1a. As shown in [23], they amount to the associated eigenvalues $\lambda_{m}$ multiplied by the characteristic wave amplitude and are given by

$$
L_{1}=\lambda_{1}\left(\frac{\partial p}{\partial x_{1}}-\rho c \frac{\partial u_{1}}{\partial x_{1}}\right)
$$




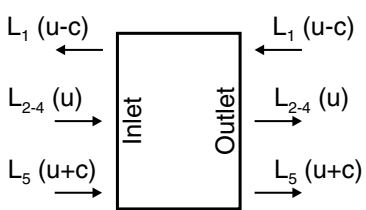

a) Full Navier-Stokes equations

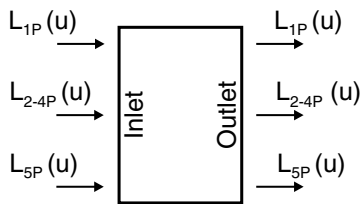

b) SICS predictor step

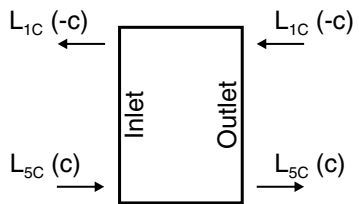

c) SICS corrector step

Fig. 1 Characteristic waves at inlet and outlet boundaries for a subsonic flow.

$$
\begin{gathered}
L_{2}=\lambda_{2}\left(c^{2} \frac{\partial \rho}{\partial x_{1}}-\frac{\partial p}{\partial x_{1}}\right) \\
L_{3}=\lambda_{3} \frac{\partial u_{2}}{\partial x_{1}} \\
L_{4}=\lambda_{4} \frac{\partial u_{3}}{\partial x_{1}} \quad \text { and } \\
L_{5}=\lambda_{5}\left(\frac{\partial p}{\partial x_{1}}+\rho c \frac{\partial u_{1}}{\partial x_{1}}\right)
\end{gathered}
$$

where $x_{1}$ is the boundary normal coordinate, and $u_{i}$ is the velocity component in the $x_{i}$ direction. Each characteristic wave $L_{m}$ is associated to a physical quantity propagated at the speed $\lambda_{m}$ through the flow. $L_{1}$ and $L_{5}$ are the acoustic pressure waves, and $L_{2}$ is the advective entropy wave, whereas $L_{3}$ and $L_{4}$ are associated with advective waves of the velocity components $u_{2}$ and $u_{3}$, respectively [23]. The fractional step method SICS involves a splitting of the acoustic and advective waves. Therefore, the characteristic waves of the predictor [Eq. (4)] and corrector [Eq. (5)] step differ from the waves of the full Navier-Stokes equations in the eigenvalues $\lambda_{m}$, whereas the wave amplitudes remain the same. The eigenvalues of the three systems of equations and the corresponding characteristic waves are shown in Fig. 1.

The characteristic waves defined by Eqs. (8-12) are substituted into the acoustic system of Eq. (5) and the continuity equation [Eq. (1)] to replace the derivatives in the $x_{1}$ direction. Using these equations, the characteristic wave equation is derived in the same way as the previously shown wave equation [Eq. ()ㅡ)]. The terms involving a derivative in boundary normal direction $\bar{x}_{1}$ of the characteristic wave equation are given by

$$
\begin{gathered}
\frac{\partial^{2} \delta p}{\partial x_{1}^{2}}=\frac{\partial}{\partial x_{1}}\left(\frac{L_{5 C}^{\delta}-L_{1 C}^{\delta}}{2 c}\right) \\
\frac{\partial u^{n+1} \delta p}{\partial x_{1}}=u_{1}^{n+1 / 2}\left(\frac{L_{5 C}^{\delta}+L_{1 C}^{\delta}}{2 c}\right)+\delta p\left(\frac{L_{5 C}^{n+1 / 2}-L_{1 C}^{n+1 / 2}}{2 \rho c}\right) \\
\frac{\partial^{2} p^{n}}{\partial x_{1}^{2}}=\frac{\partial}{\partial x_{1}}\left(\frac{L_{5 C}^{n}-L_{1 C}^{n}}{2 c}\right) \\
\frac{\partial^{2} p^{*}}{\partial x_{1}^{2}}=\frac{\partial}{\partial x_{1}}\left(\frac{L_{5 C}^{*}-L_{1 C}^{*}}{2 c}\right) \\
\frac{\partial \rho u^{*}}{\partial x_{1}}=\frac{L_{5 C}^{*}+L_{1 C}^{*}}{2 c^{2}} \text { and }
\end{gathered}
$$$$
\frac{\partial \rho u^{n}}{\partial x_{1}}=\frac{1}{c^{2}}\left(L_{2}^{n}+\frac{1}{2}\left(L_{5}^{n}+L_{1}^{n}\right)\right)
$$

where the time level $\delta$ (e.g., $L_{m}^{\delta}$ ) is computed as the difference between the waves at new and advected time levels:

$$
L_{m}^{\delta}=L_{m}^{n+1}-L_{m}^{*}
$$

Substituting Eqs. (13-18) into Eq. (6) gives the characteristic wave equation, which is solved at boundaries according to the NSCBC method.

The key advantage of the characteristic equation is the ability to compute the characteristic waves crossing a boundary separately. Following characteristics theory [24], outgoing waves are computed from inner points because information propagates out of the computational domain. Incoming waves are either computed from known information about the outside of a domain or they have to be approximated [23]. Among others, incoming waves are sent in to avoid a drift in mean flow values (e.g., to apply the far-field pressure at an outlet boundary). In this scope, Poinsot and Lele [13] proposed setting the incoming wave amplitude to

$$
\begin{gathered}
L_{\text {in }}=K\left(p-p_{\infty}\right) \quad \text { with } \\
K=\sigma c\left(1-M a^{2}\right) / l
\end{gathered}
$$

Applying the incoming wave defined by Eq. (20) reduces the drift of mean values with increasing relaxation coefficient of the boundary condition (i.e., with increasing $K$ ). As shown by Selle et al. [14], this results in a first-order filter, whose analytical reflection factor reads

$$
R=\frac{-1}{1-j(2 \omega / K)}
$$

\section{B. Time-Domain Impedance Boundary Conditions}

Higher-order filters can be applied in a CFD calculation by means of TDIBCs $[15,16]$. However, a time-domain implementation of impedance boundary conditions requires a transformation of the desired frequency response into time-space. Because an inverse Fourier transform at each numerical time step would be too costly, analytical models representing the impedance in frequency space have been proposed $[15,16,25-27]$. Because the convolution of these models is known, the boundary values can be computed directly in time-space. In this work we follow Özyörük et al. [25], who proposed a rational function as analytical model for the frequency response, which reads

$$
\begin{gathered}
H(z)=\frac{Y(z)}{X(z)} \\
=\frac{b_{0}+b_{1} z^{-1}+\ldots b_{M} z^{-M}}{1+a_{1} z^{-1}+\ldots a_{N} z^{-N}}
\end{gathered}
$$

where $z=e^{j \omega}$. Using the time-shifting property of the $z$ transform [28], the frequency response is transformed into time-space to form a difference equation as shown in $[\underline{16}, \underline{22}, \underline{25}]$ :

$$
y^{n}=-\sum_{k=1}^{N} a_{k} y^{n-k}+\sum_{k=0}^{M} b_{k} x^{n-k}
$$




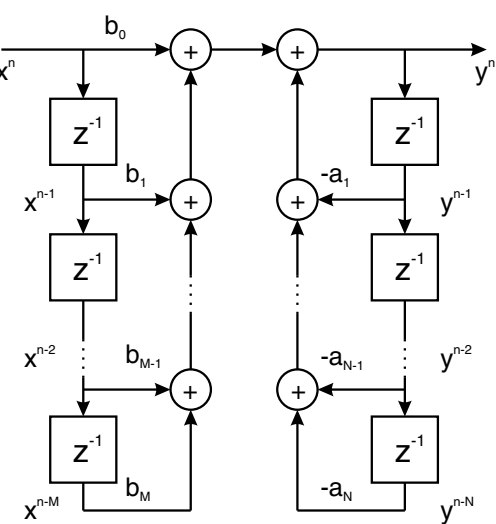

a) Direct form $I$
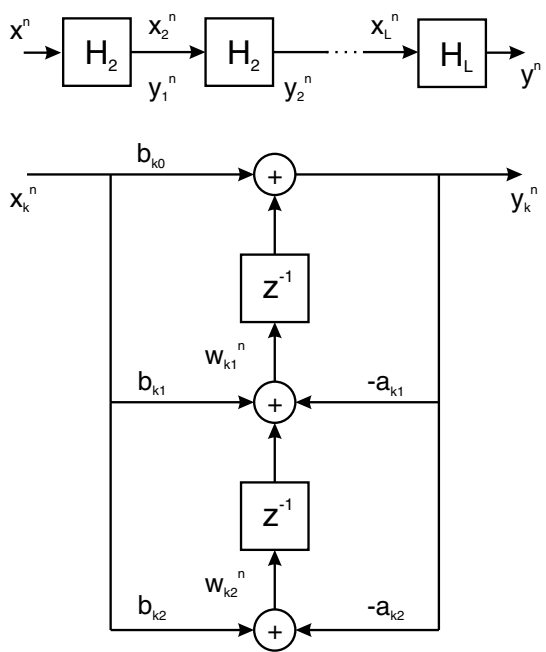

b) Direct form II transposed

Fig. 2 Filter realization as direct form I and direct form II transposed [1, 28 $]$.

Equation (25) can be involved directly in a CFD simulation that results in a "direct form I" realization [28] of the infinite-impulseresponse filter displayed in Fig. 2a. However, this realization is prone to instabilities due to roundoff errors and errors in parameter quantification [28]. Therefore, Huber et al. [16] proposed using the more stable "direct form II transposed" realization (Fig. 2b):

$$
H(z)=\prod_{k=1}^{K} H_{k}(z)
$$

where $H_{k}$ is the second-order subsystem defined by

$$
H_{k}(z)=\frac{b_{k 0}+b_{k 1} z^{-1}+b_{k 2} z^{-2}}{1+a_{k 1} z^{-1}+a_{k 2} z^{-2}}
$$

The direct form II transposed and direct form I are equivalent realizations of the frequency response $H(z)$. Using the time-shifting and convolution properties of the $z$ transform [28], the second-order subsystem [Eq. (27)] is transformed into time-space, which results in

$$
\begin{gathered}
y_{k}^{n}=b_{k 0} x_{k}^{n}+w_{k 1}^{n-1} \\
w_{k 1}^{n}=b_{k 1} x_{k}^{n}-a_{k 1} y_{k}^{n}+w_{k 2}^{n-1} \text { and } \\
w_{k 2}^{n}=b_{k 2} x_{k}^{n}-a_{k 2} y_{k}^{n}
\end{gathered}
$$

Because of better stability properties $[16,28]$, impedance boundary conditions are implemented in the DLR THETA code as direct form II transposed realization.

In the scope of CFD simulations, two different sets of input-output variables can be used to determine the acoustic boundary conditions by means of the filters discussed previously. The first option is to set the acoustic variables $p^{\prime}$ and $u^{\prime}$, which results in an impedance formulation as frequency response:

$$
\begin{gathered}
H(z)=Z(z) \\
=\frac{\hat{p}}{\hat{u}}
\end{gathered}
$$

The second option is to set the wave amplitudes $\alpha^{+}$and $\alpha^{-}$at the boundaries defined by

$$
\begin{aligned}
& \alpha^{+}=\frac{1}{2}\left(p^{\prime}+u^{\prime} \rho c\right) \\
& \alpha^{-}=\frac{1}{2}\left(p^{\prime}-u^{\prime} \rho c\right)
\end{aligned}
$$

which gives a reflection factor form as frequency response, i.e.,

$$
H(z)=R(z)
$$

$$
=\frac{\hat{\alpha}^{-}}{\hat{\alpha}^{+}}
$$

Fung et al. [29,30] proposed using the reflection factor formulation to prevent numerical instabilities. However, for CFD applications, the superiority of one of the two possible choices has not been finally assessed $[15,16]$. In this work, we follow the approach of Fung et al. $[29,30]$ and use the reflection factor to define the acoustic boundary conditions.

The computational cost to predict the boundary values with TDIBC are higher than with the NSCBC approach discussed previously. This results from the higher order of the TDIBC method, which requires including historical data, i.e., updating the state variables defined by Eqs. (28-30) at each time step. The NSCBC approach does not require the use of any data other than the values of the current time step. The higher the order of the TDIBC filter is, the more state variables have to be considered, which increases the computational costs. However, the costs are comparably low for both the NSCBC and the TDIBC approach. Regarding the computations of the EWG experiment presented in this work, no difference in the simulation runtime is observed for the different boundary models.

\section{Entropy Wave Generator Test Case}

The generation of indirect combustion noise is analyzed by Bake et al. [7] by means of the generic Entropy Wave Generator (EWG) test case. Figure 3 shows a sketch of the test rig, which is basically a convergent-divergent nozzle flow. Entropy modes are generated by heating wires located upstream of the convergent-divergent nozzle. These entropy modes are convected and accelerated through the nozzle, which gives rise to pressure fluctuations upstream and downstream of the nozzle.

Table 1 summarizes the geometrical dimensions of the analyzed test case. All axial positions are measured from the most downstream heating wire. Further details are given by Bake et al. [7]. Temperature fluctuations are measured by means of a bare wire thermocouple and 


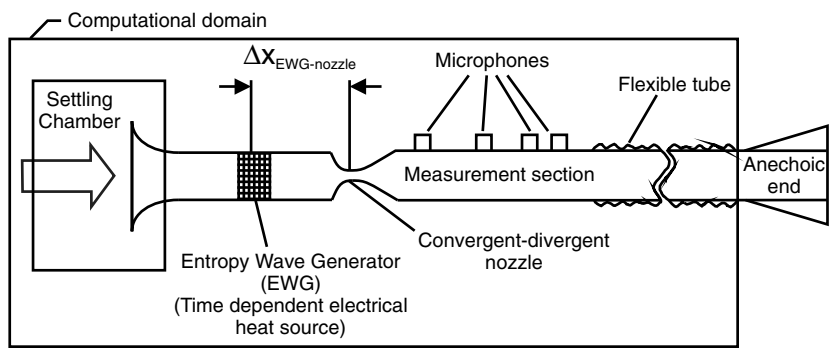

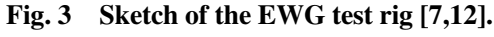

a vibrometer located between the heating module and the nozzle. Moreover, pressure fluctuations are measured at four positions downstream of the nozzle.

Bake et al. [7] defined two reference test cases of the EWG experiment. The corresponding parameters are summarized in Table 2 . The two reference test cases vary, among other parameters, in nozzle Mach number and in the heating excitation. For case 1, the nozzle throat is chocked, and four heating wires are turned on simultaneously, whereas for case 2 , a subsonic nozzle mach number is observed and six heating wires are turned on one after the other in accordance to the mean convective time between the wires. The later heating process gives rise to a sharp entropy wave with a maximum temperature fluctuation of $13.4 \mathrm{~K}$.

\section{A. Numerical Setup}

For this discussion, numerical simulations with the DLR THETA code are carried out [17]. Because of the rotational symmetry of the test rig, only a $10 \overline{\mathrm{deg}}$ slice of the flow is simulated. This simplification does not reduce the accuracy of the acoustic data gained from the simulations as shown in $[9,12]$. The slice is discretized into around 125,000 grid points, and the corresponding computational domain is displayed by Fig. 3. All transport equations are spatially discretized by means of a second-order quadratic upwind scheme. The second-order Crank-Nicolson scheme is applied as temporal discretization. Turbulent fluctuations are modeled by means of the $k-\omega$ SST turbulence model [31]. Acoustic perturbations at frequencies lower than $3 \mathrm{kHz}$ are spatially resolved with at least 50 points per wavelength. Unsteady computations are carried out at an acoustic CFL number of around 25. This leads to a time step of $\Delta t=10^{-6} \mathrm{~s}$ and hence to a maximum frequency that is temporally resolved within the computations of $500 \mathrm{kHz}$.

Table 1 Dimensions of the EWG experiment [7]

\begin{tabular}{lcc}
\hline \hline Device & Axial position, $\mathrm{mm}$ & Diameter, $\mathrm{mm}$ \\
\hline Thermocouple & 34 & \\
Vibrometer & 47.5 & \\
Nozzle throat & 105.5 & 7.5 \\
Microphone 1 & 456 & \\
Microphone 2 & 836 & \\
Microphone 3 & 1081 & \\
Microphone 4 & 1256 & \\
\hline
\end{tabular}

Table 2 Parameters of the EWG reference test cases [7]

\begin{tabular}{lcc}
\hline \hline Parameter & Case 1 & Case 2 \\
\hline Mass flow rate, $\mathrm{kg} / \mathrm{h}$ & 42 & 37 \\
Nozzle Mach number & 1.0 & 0.7 \\
Temperature increase & 9.1 & 13.4 \\
$\Delta T, \mathrm{~K}$ & & 138.2 \\
$\begin{array}{l}\text { Heating power } \\
\text { determined by } \Delta T, \mathrm{~W}\end{array}$ & 106.8 & $\begin{array}{c}\text { Numbers 1-6 delayed } \\
\text { Heated wire rings }\end{array}$ \\
$\begin{array}{l}\text { Numbers 3-6 } \\
\text { simultaneously }\end{array}$ & \begin{tabular}{c} 
according to flow velocity \\
\hline
\end{tabular} \\
\hline \hline
\end{tabular}

\section{B. Impedance of the Downstream Termination}

The computational domain is truncated downstream before the anechoic end termination of the test rig as shown in Fig. 3. The modeling of the acoustic impedance at this boundary is of crucial importance for gaining accurate results $[7,9,12]$. Leyko et al. [9] and Mühlbauer et al. [12] showed that the $E \bar{W} \bar{G}$ downstream impedance can be modeled by means of characteristic boundary conditions (NSCBCs) [13]. However, both authors proposed very different parameters to set up the boundary conditions because they had different target functions for their optimization. Leyko et al. [9] optimized the coupling parameter to minimize the least-square error between experimental and analytical modulus of the reflection factor. This leads to a relaxation coefficient of Eq. (21) that amounts to $\sigma=0.46$. On the other hand, Mühlbauer et al. [12] used a value of $\sigma=1.8$, which gives the lowest error regarding the maximum pressure fluctuation of the supersonic reference test case (case 1).

Figure 4 displays the modulus and the phase shift of the downstream reflection coefficient shifted to $x=2.325 \mathrm{~m}$, which is the boundary of the numerical domain. At signals below $100 \mathrm{~Hz}$, the modulus of the NSCBC reflection coefficient with $\sigma=0.46$ agrees very well with the experimental data, whereas at $150 \mathrm{~Hz}$ a coupling parameter of $\sigma=1.8$ results in a better agreement. Regarding the phase shift, the NSCBC with $\sigma=0.46$ agrees better with the experimental results compared to the $\sigma=1.8$ setting.

As part of this work, impedance boundary conditions (TDIBCs) are used to incorporate the experimentally measured reflection coefficient within the CFD simulations. In this scope, the coefficients of the general transfer function $H(z)$ [Eq. (23)] must be fitted to reproduce the experimental data. The bandwidth of the fitted impedance has to match the temporally resolved bandwidth, which amounts to $f \leq 500 \mathrm{kHz}$, as discussed previously. Because the most dominant pressure signals of the EWG experiment are visible at frequencies up to $200 \mathrm{~Hz}$, the downstream impedance has also only been measured in this frequency range [7]. The discrepancy between experimental and fitted bandwidth significantly reduces the accuracy of the impedance fit in the frequency range of interest. To circumvent this issue, the sampling rate at the boundaries is reduced by a fixed downsampling factor $F_{s}=25$, i.e., the state variables of Eqs. (29) and (30) are updated at each 25th computational time step. Consequently, the reflection factor is only fitted over a reduced bandwidth of $f \leq 20 \mathrm{kHz}$, which increases the accuracy of the fit.

The order of the reflection coefficient is set to $M=15$ and $N=9$ according to the ansatz function [Eq. (23)]. The corresponding model coefficients are computed by a damped Gauss-Newton method [32]. As an initial guess of the Gauss-Newton method, a complex curve fitting algorithm is applied, which seeks for the best model coefficients in a least-square sense [33]. The full algorithm is implemented in MathWorks Matlab. Figure 4 shows the fitted and measured reflection factors. The modulus of the fitted reflection factor agrees well with the measured data. However, only a reasonable agreement is visible for the phase shift. A better fit of the phase shift while retaining the accuracy of the modulus could not be computed by means of the algorithm described previously. Even increasing the order of the ansatz function does not result in a lower error regarding the phase shift.

As noted previously, the main issue for obtaining an accurate impedance fit for the EWG test case is the disagreement between the numerically resolved frequency range of $500 \mathrm{kHz}$ and the frequency range of interest of around $200 \mathrm{~Hz}$. The impedance fit covers the whole resolved frequency range, even though only signals below $200 \mathrm{~Hz}$ are dominant. To lessen this issue, a downsampling factor $F_{s}=25$ is used, which reduces the numerically resolved frequency range to $20 \mathrm{kHz}$ and increases the accuracy of the fit. Further improvement of the fit would be possible by either increasing the downsampling factor or the numerical time step. A higher downsampling factor, however, decreases the accuracy of the numerically predicted impedance at boundaries, and a higher numerical time step reduces the accuracy of the numerical solver. Therefore, the impedance fit shown in Fig. $\underline{4}$ is used for this discussion. 


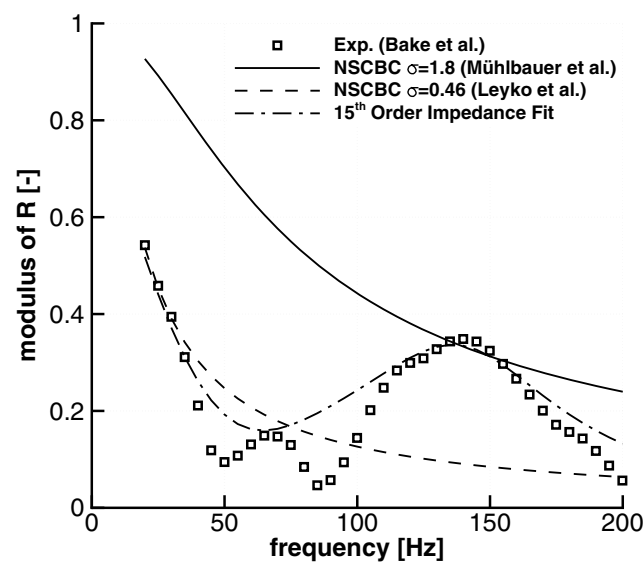

a) Modulus

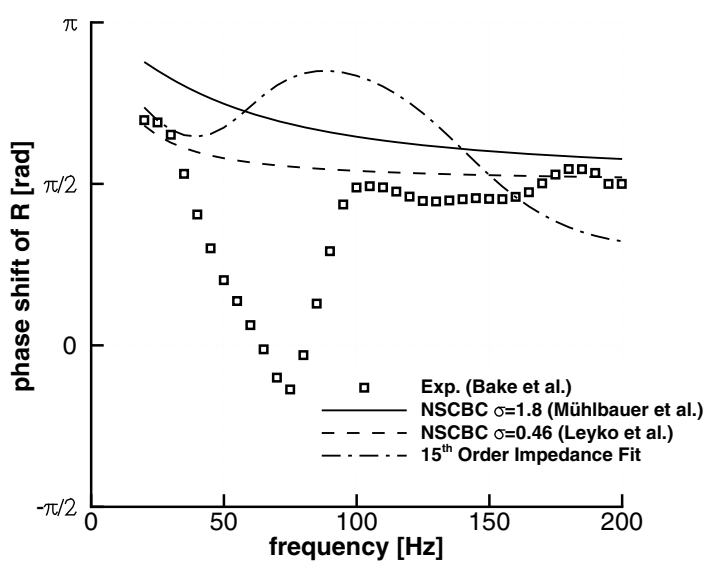

b) Phase shift

Fig. 4 Reflection factor of the EWG downstream termination.

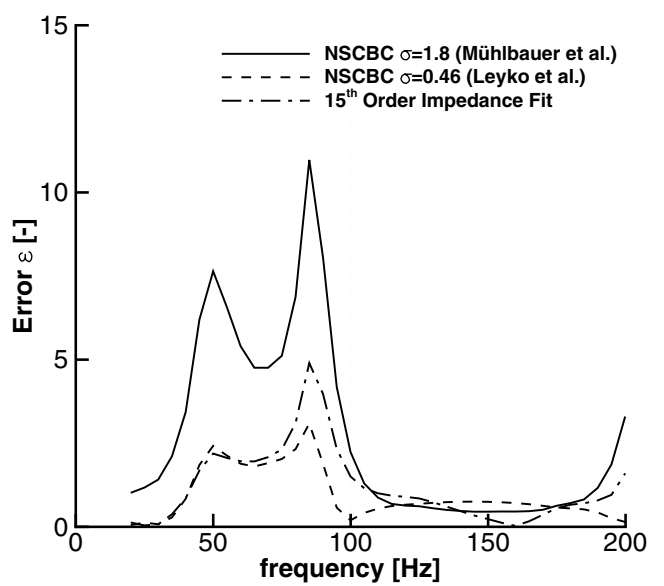

Fig. 5 Error of the downstream impedance approximations.

To evaluate the accuracy of the impedance fit, the error $\epsilon$ between fitted and experimental reflection factor,

$$
\epsilon=\frac{\left|R-R_{\text {exp }}\right|}{\left|R_{\text {exp }}\right|}
$$

is plotted in Fig. 5. The largest error is computed for the NSCBC with a coupling parameter of $\sigma=1.8$, which amounts to around 12 at $90 \mathrm{~Hz}$. Comparing the errors plotted for NSCBC with $\sigma=0.46$ and the 15th-order impedance fit shows that both boundary conditions result in almost equal errors. A higher accuracy is achieved by the NSCBC at around $90 \mathrm{~Hz}$, whereas the error of the impedance fit is lower at around $150 \mathrm{~Hz}$. Even though the error computed for the NSCBC with $\sigma=0.46$ and the impedance fit are almost equal, using the latter results in a higher agreement between numerical and experimental data, as discussed in Sec. $\underline{V}$. This might be caused by the good agreement in the modulus of the reflection factor between the 15th-order impedance fit and the experimental data (Fig. 4). The data measured for the EWG experiment are strongly dominated by low-frequency signals. This lessens the impact of an inaccurate approximation of the phase shift applied at the downstream termination of the test rig.

\section{Heating Excitation}

Within the EWG experiment, entropy modes are generated by heating wires upstream of the nozzle [7]. For CFD calculations, this heating process is implemented as sources of the energy equation. The source distribution is commonly computed as product of a temporal evolution $\zeta(t)$ and a spatial distribution $\psi(x)[\underline{9}, \underline{10}, \underline{12}]$, i.e.,

$$
\Phi(x, t)=\Phi_{0} \zeta(t) \psi(x)
$$

For the supersonic EWG reference case 1 (Table 2), Mühlbauer et al. [12] proposed modeling the temporal source evolution as a linear increase and exponential decrease in time. However, especially the initial increase of the source term can be modeled very accurately by an exponential ansatz function. Therefore, Leyko et al. [9] proposed modeling both heating processes as exponential evolutions, i.e.,

$$
\zeta(t)= \begin{cases}0, & \text { if }<t_{0} \\ 1-e^{-\left(t-t_{0}\right) / \tau}, & \text { if } t_{0} \leq t \leq t_{0}+t_{P} \\ \zeta\left(t_{0}+t_{p}\right) e^{-\left(t-t_{0}\right) / \tau}, & \text { if } t>t_{0}+t_{P}\end{cases}
$$

This approach is also used by Duran et al. [10] with $\tau=7 \mathrm{~ms}$ to model the heat sources of the subsonic EWG reference case 2 . Figure 6a shows the experimentally gained temperature evolution downstream of the heating wires for the subsonic EWG case 2 and the exponential approach defined by Eq. (39) for different relaxation coefficients. Minimizing the least-square error between the experimental data and the exponential model function for the heating-up regime $(t \leq 0.2 \mathrm{~s})$ leads to a relaxation of around $\tau=3.5 \mathrm{~ms}$. As visible in Fig. 6a, this setting results in a very good agreement between experimental and model data. Therefore, the model function [Eq. (39)] is used for this work.

Besides the temporal evolution, the spatial distribution of the computational energy sources is also modeled. Leyko et al. [9] proposed approximating the spatial distribution as a hyperbolic tangent. However, within the experiments, the flow is heated up by wire rings at discrete $x$ locations [7]. Therefore, Mühlbauer et al. [12] put a discrete spatial source distribution forward, which reads

$$
\psi(x)= \begin{cases}1 & \text { if }\left|x_{h, i}-x\right|<\frac{\Delta x}{2} \\ 0 & \text { else }\end{cases}
$$

Figure $6 \mathrm{~b}$ displays the discrete source distribution described by Eq. (40). This discrete distribution is closer to the experimental setup and hence more accurate. This applies especially for calculations of the EWG reference case 2, where the powering of the different heating wires is delayed by the convective time between the wires. This control sequence of the heating wires is implemented for this discussion by delaying the source function [Eq. (38)] at each discrete source location. Using the hyperbolic tangent function as the spatial distribution of the heat sources as proposed in [9] does not allow for a delay time between the heating wires.

\section{Direct Noise Model}

The EWG experiment was set up by Bake et al. [ㄱ, $\underline{8}]$ to investigate the generation of indirect noise. However, recent analytical results by Duran et al. [10] propose that the ratio of indirect to direct noise amounts to around 0.01 within the subsonic EWG experiment, and hence the noise signal measured is mainly caused by direct noise. 


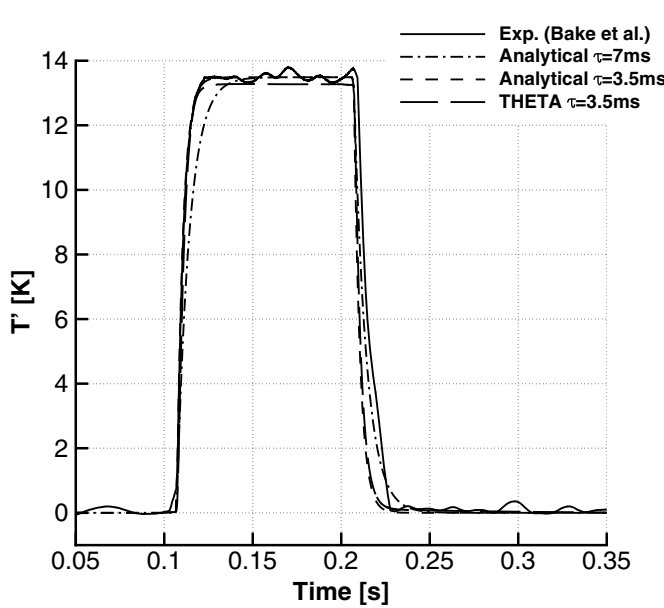

a) Temporal

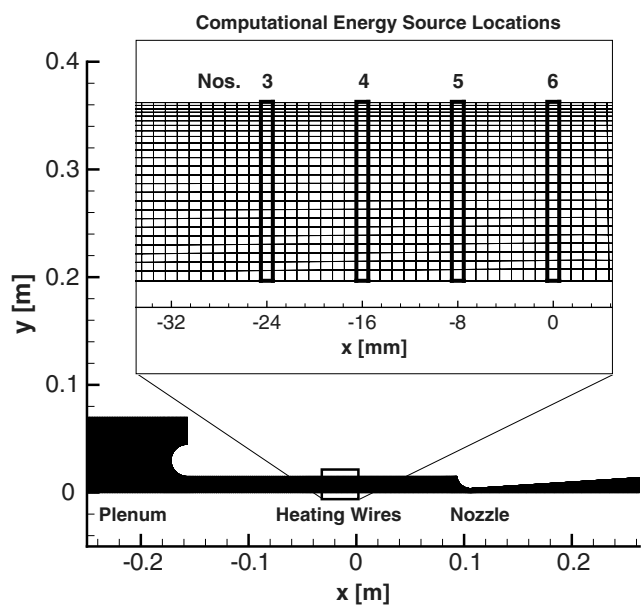

b) Spatial

Fig. 6 Temporal and spatial distribution of the energy sources.

Furthermore, Giauque et al. [11] also presented analytical results, which indicate that the temperature fronts generated within the subsonic EWG experiment are not steep enough to cause the measured pressure fluctuations through an indirect noise mechanism.

Direct noise is generated within the EWG experiment at the locations of the heating wires (i.e., upstream of the nozzle). The direct noise is then transferred through the nozzle and finally measured downstream of the nozzle [10]. To evaluate the contribution of direct noise numerically, the heat sources upstream of the nozzle are replaced within this work by acoustic sources at the same locations. Acoustic sources do not generate entropy waves, and hence the indirect noise signal is removed from the test case. This allows computing only the direct noise contribution measured within the EWG experiment.

As discussed in Sec. II, a fractional step method based on characteristic splitting of the Navier-Stokes equations is used in this work. The second fractional step, i.e., the pressure correction [Eq. (6)], involves only the acoustic modes of the flow. Acoustic modes are therefore generated by introducing a source term:

$$
\Psi(x, t)=\frac{4}{c^{2} \Delta t^{2}}\left(p(x, t)-p_{s}(x, t)\right)
$$

in the pressure correction equation, which is in line with the time derivative term of this equation, where $p$ and $p_{s}$ are the predicted and the specified pressure values, respectively. The source term is only applied at the locations of the heating wires. To ensure that the strength of the acoustic and the heat sources are equivalent, the specified pressure $p_{s}$ is set to the pressure signal generated by the EWG heat sources at the locations of the heating wires. The pressure generated by the EWG heat sources in turn is gained from the numerical calculations with the heat source model discussed in the previous section.

Figure $7 \mathrm{a}$ displays the acoustic wave amplitudes gained at $x=-100 \overline{\mathrm{mm}}$, which is located between the upstream settling chamber and the heating wires (Fig. $6 \mathrm{~b}$ ). The wave amplitudes of direct noise are computed by means of acoustic sources that are of the strength of the EWG heat sources. They agree very well with the wave amplitudes predicted for EWG noise, which are computed from the heat source model discussed in the previous section. This shows that the sources used for the computation of direct noise have the same acoustic strength as the EWG heat sources.

Figure $7 \mathrm{~b}$ shows the wave amplitudes at the location of the vibrometer (i.e., between the heating wires and the nozzle). To compute the wave amplitudes, a constant mean flow is assumed. However, within the EWG experiment an entropy mode is convected through the location of the vibrometer, which alters the mean flow. Starting from a steady state, the flow is heated up and accelerated at time $t \approx 0.11 \mathrm{~s}$ and reaches a new approximately steady state at $t \approx 0.125 \mathrm{~s}$, as shown by the temperature profile plotted in Fig. 6 a. At time $t \approx 0.2 \mathrm{~s}$, the flow is again cooled down and decelerated to the initial steady state. The wave amplitudes can only be computed during the steady states of the flow, as shown in Fig. $7 \mathrm{~b}$ for the THETA results with the EWG heat sources. Vice versa, at the times of unsteady mean flow the wave amplitudes are not plotted for the computations with entropy modes. In contrast, the mean flow is not

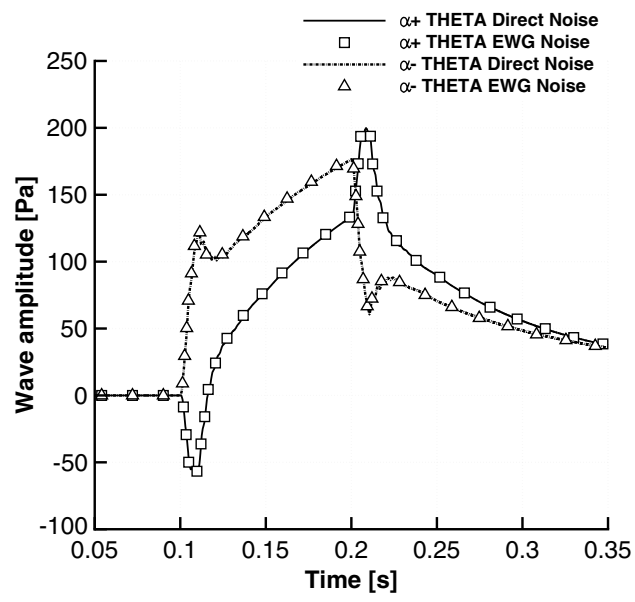

a) $x=-100 \mathrm{~mm}$

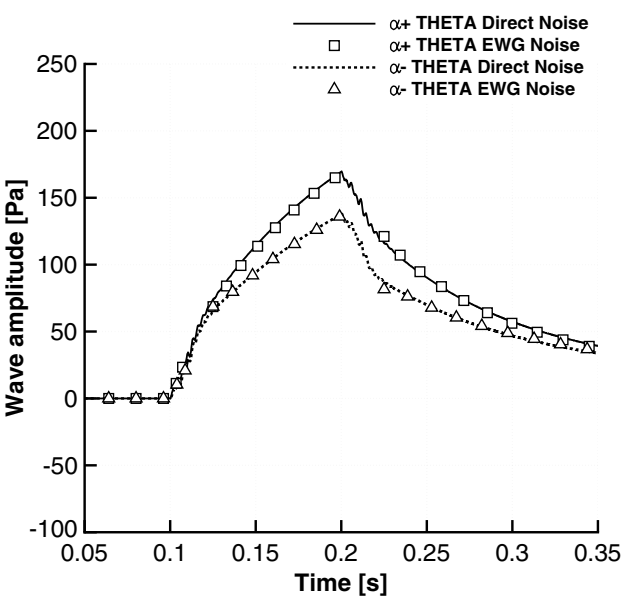

b) $x=47.5 \mathrm{~mm}$ (vibrometer)

Fig. 7 Acoustic wave amplitudes between the settling chamber and the nozzle. 


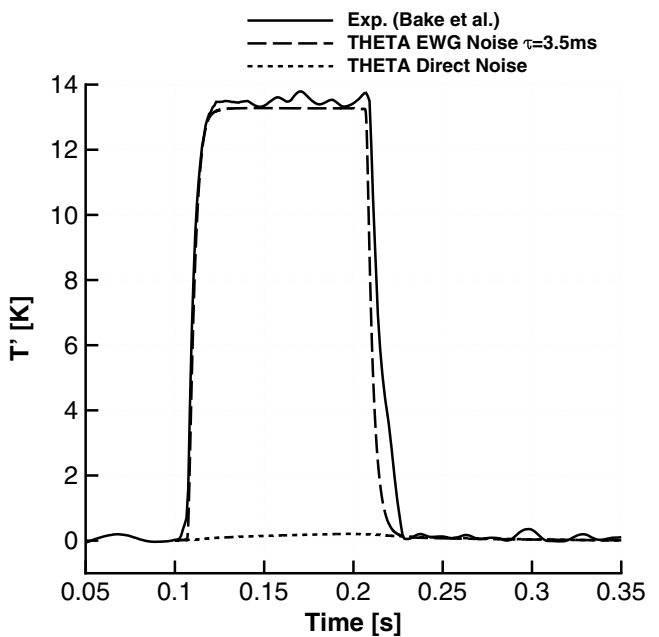

Fig. 8 Temperature signal at the vibrometer.

altered in case of the direct noise computations. Therefore, the wave amplitudes are visible for the whole time period shown in Fig. $7 \mathrm{~b}$ for the THETA results of direct noise.

The acoustic waves predicted for direct and EWG noise agree very well, as presented in Fig. 7b. During unsteady mean flow, the waves cannot be compared at this position. However, a significant difference between the waves would also be visible at the more upstream position $x=-100 \mathrm{~mm}$ displayed in Fig. 7a. The waves agree very well for all times at $x=-100 \mathrm{~mm}$, which shows that the acoustic sources generate the same pressure waves upstream of the nozzle as the EWG heat sources. The wave propagating in positive $x$ direction $\alpha^{+}$at the location of the vibrometer is partially reflected at the nozzle and partially transferred through the nozzle. The latter part causes the direct noise signal measured within the EWG experiment [10]. Because the pressure waves upstream of the nozzle are equal for direct and EWG noise, the direct noise predicted downstream of the nozzle is also equal in both simulations.

Figure 8 presents the temperature profiles at the location of the vibrometer. The profile predicted for EWG Noise is in accordance with the experimental data. Considering the numerical data from computations for only direct noise shows that the flow temperature is not increased because no entropy mode is generated by the sources. Consequently, no entropy noise is present in this computation.

\section{Results}

One of the major results of the EWG investigations is the time series of pressure fluctuations measured within the measurement section downstream of the nozzle (Fig. 3). The pressure signal obtained for the subsonic EWG case 2 (Table 2 ) is used in this section to discuss the accuracy of the models used for the heat sources and the downstream impedance termination. In this scope, zones of influence of the different models on the time series of pressure signals are defined initially, before the models are assessed. Finally, numerical results for indirect and direct noise are presented and compared to experimental data.

\section{A. Time Delays of Direct and Indirect Noise}

Within the EWG experiment, entropy modes are generated by heating wires upstream of the nozzle (Fig. 3). These modes are convected with flow velocity $u$ through the nozzle, which induces indirect noise. By contrast, direct noise is generated by the heating

Table 3 Time delays of direct and indirect noise at microphone 4

\begin{tabular}{lcc}
\hline \hline Noise source & First signal & First reflected signal \\
\hline Direct & $3.56 \mathrm{~ms}$ & $9.76 \mathrm{~ms}$ \\
Indirect & $12.65 \mathrm{~ms}$ & $18.86 \mathrm{~ms}$ \\
\hline \hline
\end{tabular}

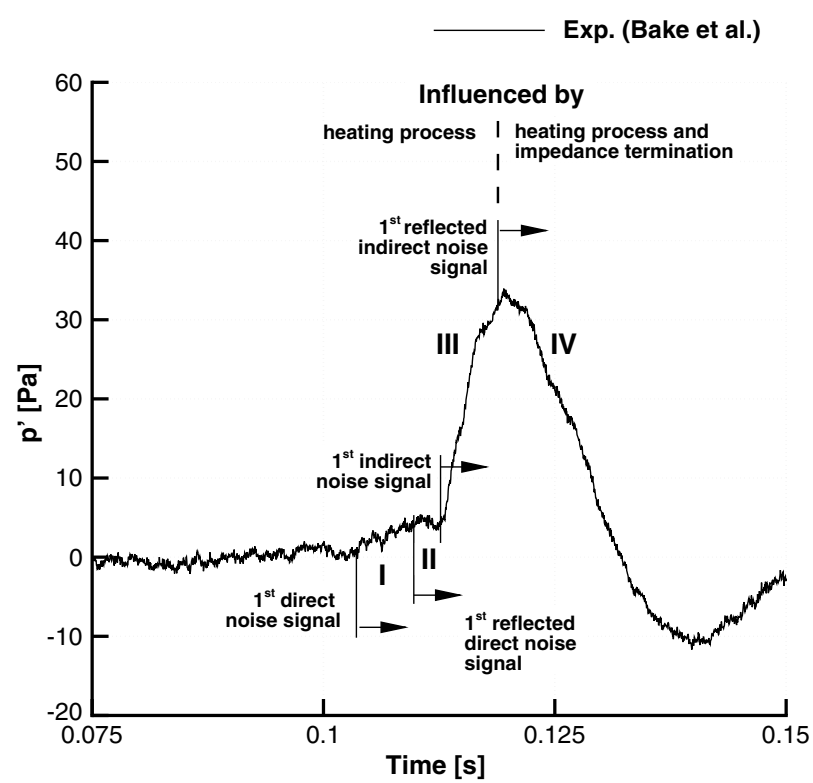

Fig. 9 Time delays of different noise signals at microphone 4.

process itself and propagates at $u \pm c$, where $c$ is the speed of sound. Because of these different propagation speeds, the first signals of direct and indirect noise are measured with different delay times within the measurement section [8]. The delay time $t_{D}$ of a signal propagated over a distance $x$ reads

$$
t_{D}(x)=\int_{x_{0}}^{x_{0}+x} \frac{\mathrm{d} x}{s(x)}
$$

As part of this discussion, Eq. (42) is solved along the centerline of the test rig by means of CFD data. Because the highest flow velocity in the $x$ direction is visible on the centerline, the computed time delays represent the respective minimum values. Table 3 presents the delay times of direct and indirect noise at microphone 4 (Table 1). The first signal of indirect noise arrives at the fourth microphone around $9 \mathrm{~ms}$ later than the first signal of direct noise. Moreover, the signals propagate further downstream and are reflected at the downstream impedance termination of the test rig. The delay times of these reflected waves are also shown in Table 3 .

Figure 9 displays the experimentally obtained pressure signal by Bake et al. [7] at microphone 4 and the previously discussed delay times at this location. The heating wires are turned on within the EWG experiment at $t=0.1 \mathrm{~s}$. Therefore, the time axis of Fig. 9 is shifted by this value compared to the delay times presented in Table 3 . The delay times are used to define four initial time sections (i.e., Secs. I-IV) of the signal. Before the first indirect noise signal is measured (I-II), a maximum increase of around $3 \mathrm{~Pa}$ compared to the undisturbed signal is visible. This increase is due to direct noise generation of the heating wires. After the first indirect noise signal arrives at the microphone, a steep rise of pressure is observed in time section III up to a maximum value of around $33 \mathrm{~Pa}$. The location of the first maximum pressure value almost coincides with the delay time of the first reflected indirect noise signal (Fig. 9).

The analysis of the delay times leads to two conclusions regarding the modeling of the heating process and the downstream boundary conditions, which were discussed previously. First, signals reflected at the downstream termination are of minor importance in time Secs. I-III. The only reflected signals originate from direct noise, but these signals are shadowed by nonreflected indirect noise. Therefore, the pressure signal gained in Secs. I-III is almost independent of the downstream impedance termination, which means, in turn, that it is mainly controlled by the heating process. Second, the superposition of the reflected indirect noise signals gives rise to a rapid change in the pressure series in time Sec. IV.

Because the pressure fluctuations measured at microphone 4 (Fig. 9) are mainly influenced by the heating process in Secs. I-III, 


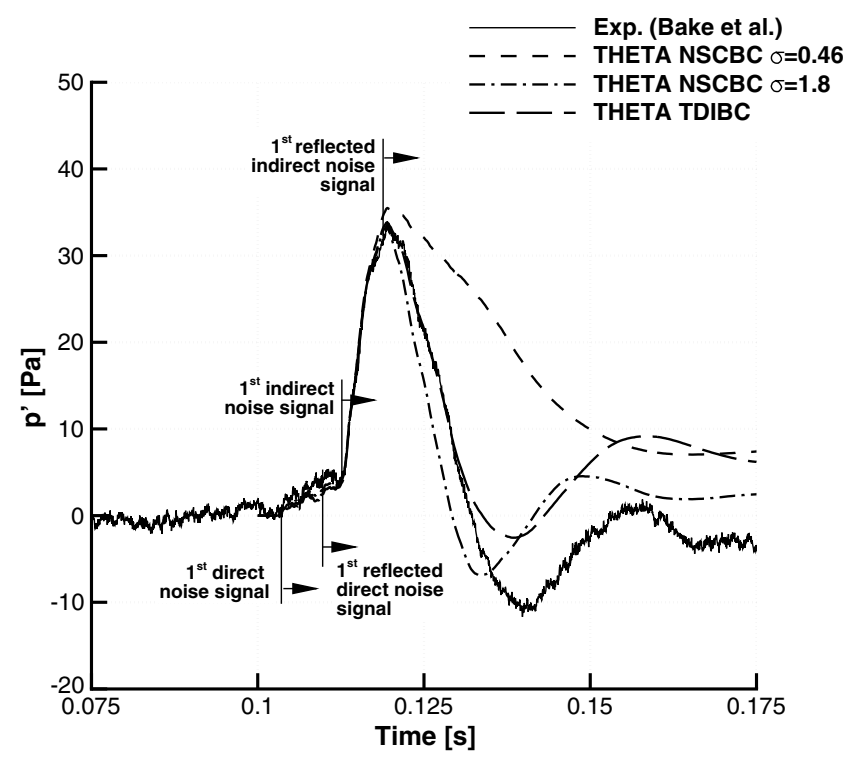

Fig. 10 Pressure series for different downstream boundary conditions.

signals within these sections are used in the following to assess the accuracy of the heating source model (Sec. V.C). Furthermore, the downstream impedance has a strong influence on the signals gained in Sec. IV. Consequently, the accuracy of the boundary conditions model is discussed by means of the pressure signals measured in this time section.

\section{B. Influence of Boundary Condition Models}

Figure 10 shows the pressure fluctuations obtained experimentally and numerically with different boundary conditions for the downstream termination of the test rig. It is observed that the downstream boundary condition only influences the pressure signal after the first reflected indirect noise signal reaches the microphone. This confirms the conclusions drawn from the delay time analysis presented in the previous subsection.

Numerical results computed with NSCBC invoking the settings $\sigma=1.8$ and $\sigma=0.46$ proposed by Mühlbauer et al. [12] and by Leyko et al. [9], respectively, are presented in Fig. 10. The computations are carried out with $\tau=3.5 \mathrm{~ms}$ for the relaxation of the temporal heat source evolution. Pressure fluctuations computed with

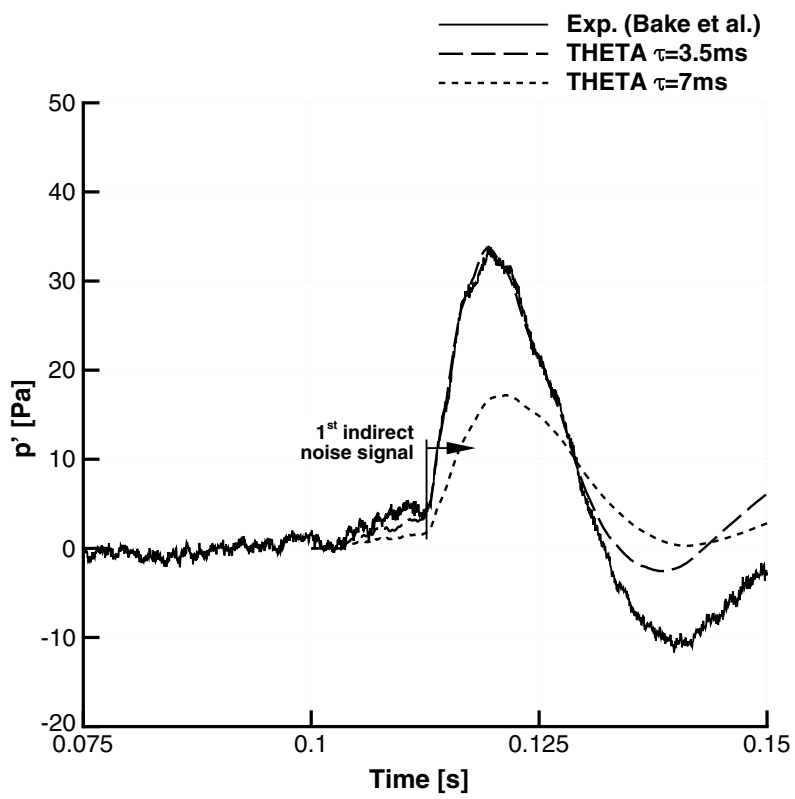

a) Relaxation factor the higher relaxation coefficient $\sigma=1.8$ agree better with the experimental data. Generally speaking, a higher modulus of the reflection factor results in a steeper decrease after the first maximum at $t \approx 0.12 \mathrm{~s}$. Therefore, the $\sigma=0.46$ underestimates the modulus of the reflection at the downstream EWG impedance termination, as shown in Fig. 4a. By contrast, using a value of $\sigma=1.8$ overestimates the modulus of the reflection.

Applying the fitted downstream impedance presented in Fig. 4a by means of the TDIBC results in a very good agreement between numerical and experimental data. The pressure decline after the first maximum is reproduced very accurately by the numerical data. The locations of the numerically computed minima and maxima also agree well with the experimental data. However, at the first pressure minimum at $t \approx 0.14 \mathrm{~s}$, a difference of around $8 \mathrm{~Pa}$ is visible. This difference might result from the error in phase shift of the fitted impedance function compared to the experimental data as discussed in Sec. IV.B.

\section{Influence of Heat Source Model}

The modeling of the heat sources commonly used for CFD calculations of the EWG is discussed in Sec. IV.C. In the following, the influence of the temporal evolution of the heat sources on the pressure signal gained at microphone 4 of the test rig is discussed. Within the previous subsections, it is shown that this pressure signal is mainly determined by the heating process until the first reflected indirect noise signal is measured (i.e., until $t \approx 0.12 \mathrm{~s}$ ). Hence, this time period is used to analyze the accuracy of the heating sources.

Numerical calculations are carried out for different temporal evolutions of the heat sources. First, different exponential relaxation coefficients $\tau$ of Eq. (39) are applied. As shown in Sec. IV.C, a leastsquare optimization of the relaxation coefficient results in a value of $\tau=3.5 \mathrm{~ms}$. In contrast, Duran et al. [10] proposed a value of $\tau=7 \mathrm{~ms}$. The respective model functions of the heat sources are plotted in Fig. 6a. Second, the heat sources are delayed by the convective time between the heating wires within the subsonic EWG experiment [7]. To assess the influence of this convective delay, calculations are carried out with and without delayed heat sources.

Figure 11 presents the time series of pressure fluctuations gained experimentally [7] and numerically. The numerical calculations are carried out with TDIBC. The impact of different relaxation factors is shown by Fig. 11a. Setting the relaxation to the optimized value $3.5 \mathrm{~ms}$ results in a very good agreement between experimental and numerical data for $t \leq 0.12 \mathrm{~s}$, as shown in the previous subsection. Increasing the relaxation to $\tau=7 \mathrm{~ms}$ generates a shallower shape of

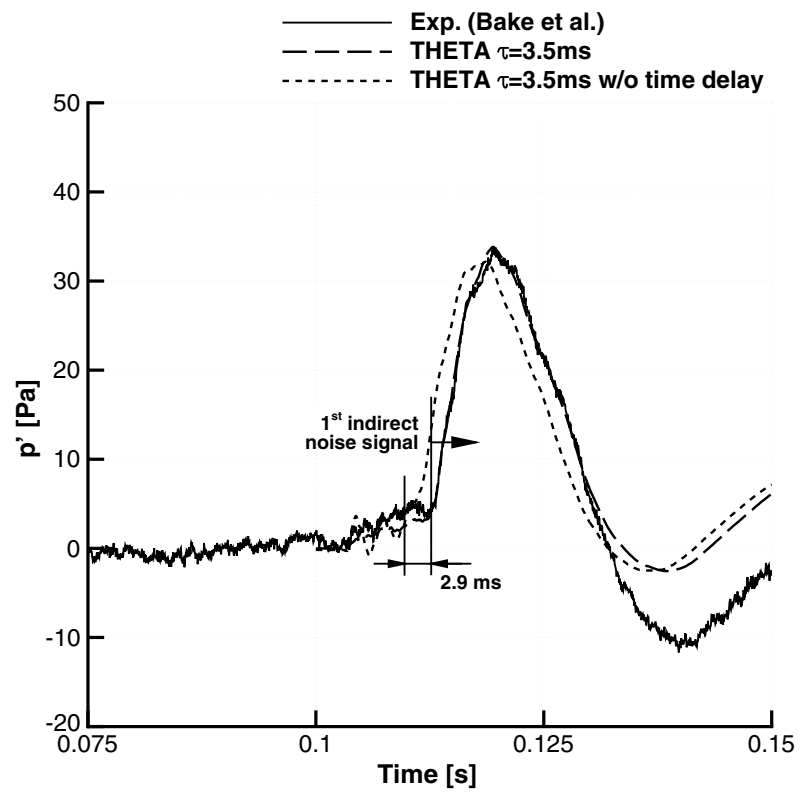

b) Time delay

Fig. 11 Impact of two model parameters on the downstream pressure signal. 


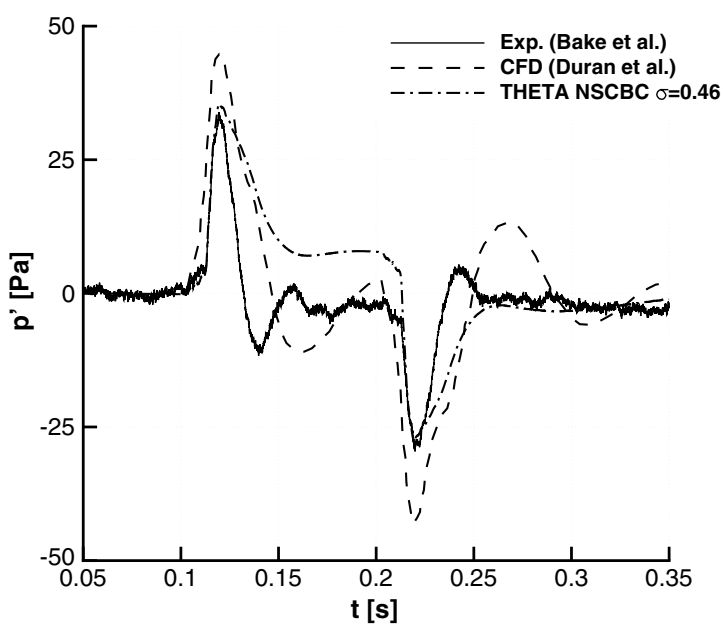

a) $\mathrm{NSCBC}$

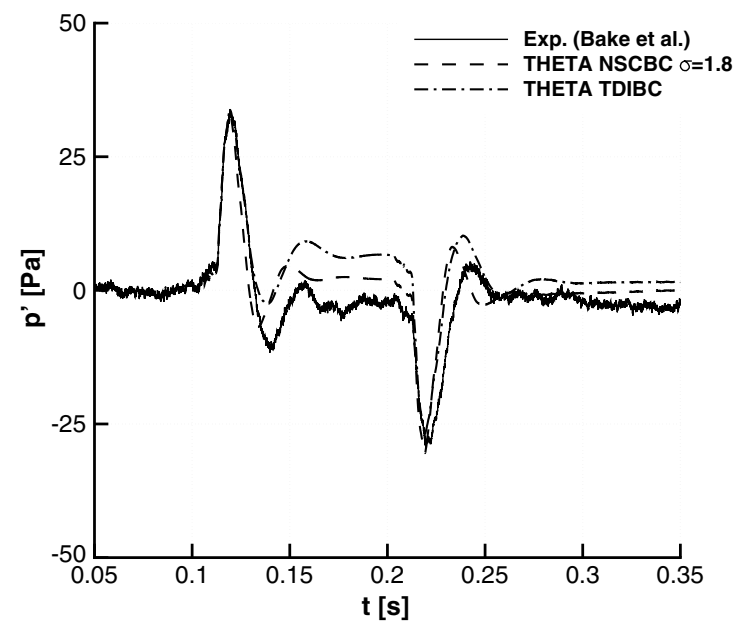

b) NSCBC and TDIBC

Fig. 12 Time series of pressure fluctuations gained for the EWG reference case 2.

the entropy wave while retaining the maximum temperature fluctuation of $\Delta T=13.4 \mathrm{~K}$ (Fig. 6 a). This leads to a less steep pressure increase before the first maximum plotted in Fig. 11a and hence to a lower maximum pressure fluctuation. This finding agrees with analytical results computed for the subsonic EWG experiment by Giauque et al. [11]. The maximum pressure fluctuation is almost reduced by half in the case of the less sharp entropy wave.

Figure $11 \mathrm{~b}$ displays the impact of the convective delay time between the heat sources applied for the subsonic EWG reference case. The numerical results from calculations with delayed heat sources agree well with the experimental data, as discussed before. In the case of synchronized sources, the heating process of the most downstream source starts around $2.9 \mathrm{~ms}$ earlier compared to the delayed source sequence. Consequently, the first indirect noise signal is obtained earlier in the measurement section. Indirect noise results in a steep rise of the pressure downstream of the nozzle, as discussed before. Therefore, the initial pressure increase visible in Fig. 11b is shifted toward lower times if the sources are not delayed by the convective times between them. Moreover, the peak pressure value is only reduced slightly by around $1.5 \mathrm{~Pa}$ compared to the simulation results with a delayed heat source control sequence.

\section{Indirect Noise}

Within the previous subsections, the accuracy of the heat source and boundary impedance models was discussed by means of the initial $50 \mathrm{~ms}$ of the pressure response of the subsonic EWG reference case (Table 2). In the following, the whole pressure signal gained from CFD calculations and experiments [7] is analyzed. Figure 12a presents the experimental and two different numerical results. Regarding the numerical data, calculations carried out for this discussion are compared to results reported by Duran et al. [10]. The pressure response computed with DLR THETA shows a better overall agreement with the experimental data and a higher accuracy regarding the maximum pressure fluctuations. Both CFD calculations are carried out with NSCBC and a relaxation of $\sigma=0.46$. However, among other things, the heat source modeling is different for both computations, possibly leading to the discrepancy of the numerical results.

Figure $12 \mathrm{~b}$ shows the time series of the pressure obtained experimentally and computed with different boundary conditions for the downstream termination. The calculations with TDIBC lead to the highest accuracy at the first maximum of the pressure fluctuations compared to the experiments of the considered boundary conditions. Furthermore, the TDIBC results show a very good agreement with experimental data regarding the locations of the minima and maxima. However, a constant offset between the TDIBC data and experiments is visible in the region $0.14 \mathrm{~s} \leq t \leq 0.2 \mathrm{~s}$. This offset is lower in the case of the NSCBC with $\sigma=1.8$ computations.
Figure 13 illustrates the power spectral density (PSD) obtained at microphone 4 . The PSDs are computed from time signals with a numerical sampling time of $35 \mathrm{~ms}$. To enhance the resolution in the lower-frequency regime and to match the experimental sampling time, the numerical data are extended by zero-padding to a sampling time of $0.9 \mathrm{~s}$, as proposed by Mühlbauer et al. [12]. The better agreement with experimental data of the computations with TDIBC compared to the NSCBC calculations is also visible in the PSD plots. In the case of NSCBC with a relaxation of $\sigma=0.46$ (Fig. 13a), the energetic content is overestimated for $f \leq 15 \mathrm{~Hz}$ and underestimated for the two following peaks. The spectral content obtained with $\mathrm{NSCBC}$ and $\sigma=1.8$ is underestimated in the low-frequency range (i.e., $f \leq 30 \mathrm{~Hz}$ ). The calculations invoking TDIBC show the best overall agreement with the experimental data in comparison with the NSCBC simulations. Especially in the frequency range with the most energetic content, the TDIBC agree well with the experimental data.

\section{E. Direct Noise}

In Sec. V.A of this work, the time delays for direct and indirect noise signals are associated with different changes of the pressure signal gained at microphone 4 of the EWG experiment (Table 1). Only a small pressure increase of around $3 \mathrm{~Pa}$ is visible before the first indirect noise signal is measured. Thereafter, the pressure rises steeply until the first reflected indirect noise signal arrives at the microphone. The point in time of the first pressure maximum agrees very well with the delay time of the first indirect noise signal reflected at the downstream termination (Fig. 9). This indicates that the pressure signal measured at microphone 4 is dominated by indirect noise generated within the nozzle rather than by direct noise generated by the heat sources upstream of the nozzle. This is further confirmed by the plots shown in Fig. 11b, which compare pressure time series for different temporal evolutions of the heat sources. In one case, the heat sources are turned on one after the other in accordance with the convective delay time between the heating wires, whereas the sources are synchronized in the other case. Synchronizing the sources reduces the time delay of the indirect noise signal. As presented in Fig. 11b, the pressure rise is accordingly shifted toward smaller times, which also indicates that this steep increase is caused by indirect noise.

The time-delay analysis, however, does not give a quantitative prediction of the direct noise contribution within the EWG experiment. Therefore, the direct noise contribution is computed as part of this work by using acoustic sources upstream of the nozzle, as discussed in Sec. IV.D. Numerical calculations are carried out with TDIBC as the downstream boundary condition and a relaxation factor of $\tau=3.5 \mathrm{~ms}$ as temporal evolution of the heat sources. This setup gives the most accurate results when compared to the experimental data. Figure 14 compares the pressure signals predicted at microphone 4 to the experimental data for direct and EWG noise. 


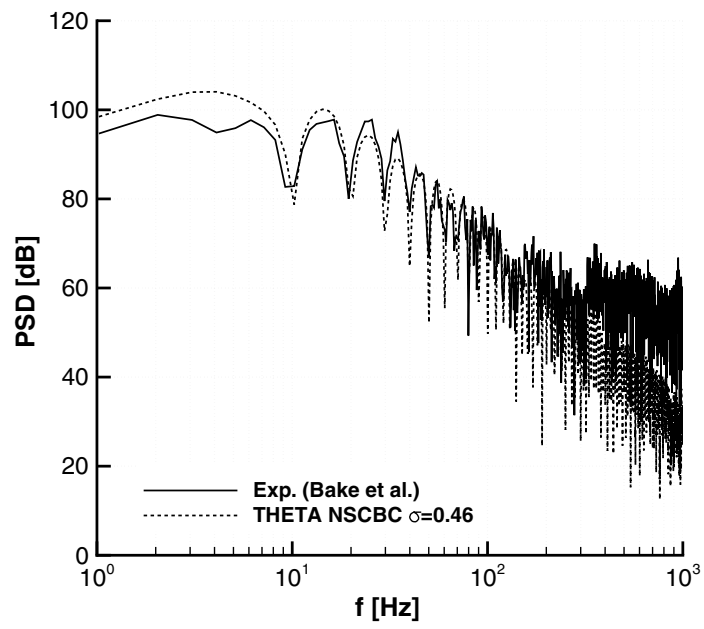

a) $\mathrm{NSCBC} \sigma=\mathbf{0 . 4 6}$

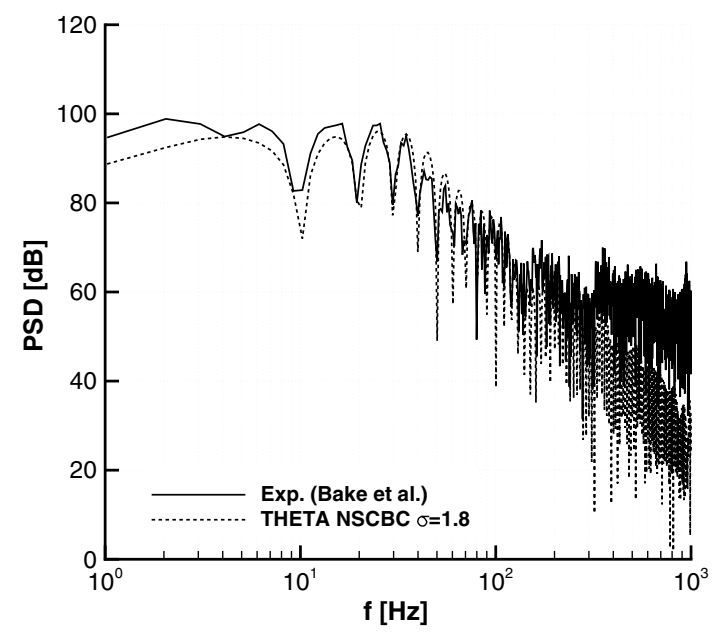

b) $\mathrm{NSCBC} \sigma=1.8$

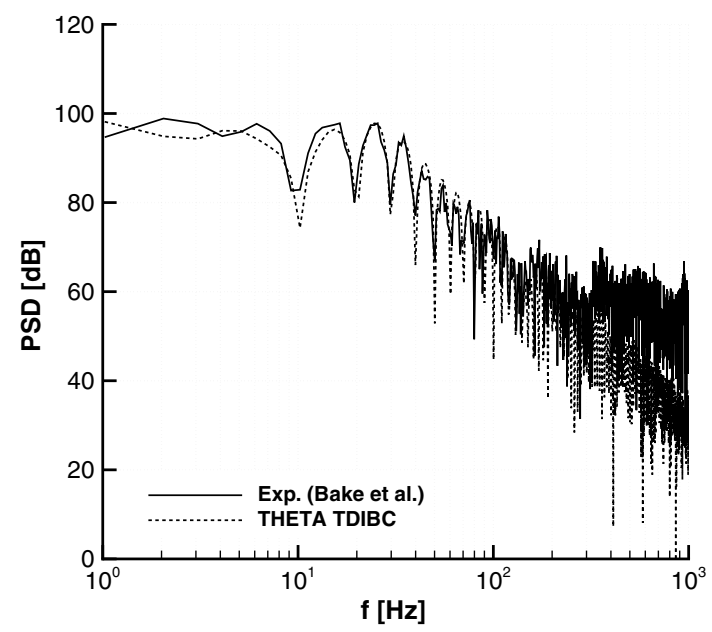

c) $15^{\text {th }}$ order TDIBC

Fig. 13 Power spectral densities gained for the EWG reference case 2.

The numerical simulation of the EWG noise (i.e., with heat sources) predicts the first pressure maximum accurately, as discussed previously. Both numerical data sets agree well until the delay time of the indirect noise. Thereafter, only the data obtained from the computation with heat sources show a steep pressure rise, which is in accordance with the experimental data. This shows that the entropy wave generated by the heat sources causes the steep pressure rise, and hence the signal is dominated by indirect rather than direct noise. Comparing the peak pressure values gained from the computations

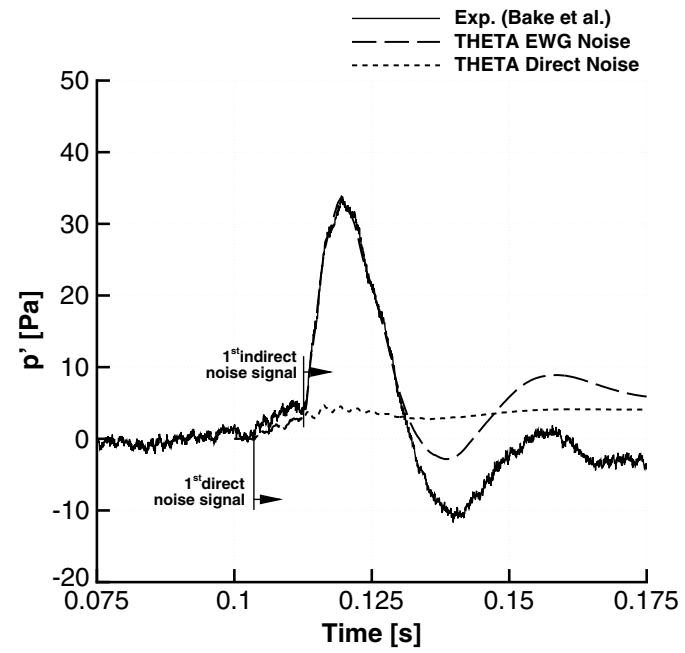

Fig. 14 Time series of the pressure at microphone 4. with acoustic and heat sources of around $5 \mathrm{~Pa}$ and $33 \mathrm{~Pa}$, respectively, shows that direct noise is not completely negligible. The direct noise amplitude is approximately 6-7 times lower than the amplitude measured within the EWG experiment. As presented in Fig. 7b, the inclining acoustic wave amplitude upstream of the nozzle is remarkably strong, with a maximum value of around $170 \mathrm{~Pa}$. Comparing this to the maximum pressure value of $5 \mathrm{~Pa}$ downstream of the nozzle reveals that only a small part of the acoustic wave is transferred through the nozzle. This might be caused by the rapid area change of the nozzle.

It should be noted, however, that the entropy wave of the EWG experiment alters the acoustic transfer behavior of the nozzle. Hence, the transfer behavior is slightly different for the simulations with and without entropy mode. The Mach number within the nozzle increases by approximately $3 \%$ due to the entropy wave. The maximum pressure fluctuation measured downstream of the nozzle depends on the nozzle Mach number. Bake et al. [7] published experimental and numerical data for different nozzle Mach numbers. Based on this data set, the change in the maximum pressure fluctuation due to an increased nozzle Mach number by $3 \%$ is estimated to be less than $1 \mathrm{~Pa}$ for the subsonic EWG case. This is much lower than the change of the peak pressure value of around $28 \mathrm{~Pa}$ discussed previously. Hence, the influence of different acoustic transfer behavior of the nozzle predicted in the simulations with and without entropy mode is neglected in this discussion.

\section{Conclusions}

The Entropy Wave Generator (EWG) experiment is a generic test case on entropy noise (i.e., on indirect combustion noise [7]). It has 
been studied extensively by experimental []] , analytical [ㄱ, $\underline{9}, 10]$, and numerical $[7,9,10,12]$ approaches. In the scope of the numerical computations, different modeling approaches of the heat sources and the boundary conditions have been reported in literature. These models were analyzed in the work at hand, and based on this, the simulations are advanced by more accurate models. Computations were carried out for the subsonic EWG reference case (case 2) and compared with the respective experimental data.

The heat sources commonly used for CFD calculations of the EWG were enhanced by combining the models put forward by Leyko et al. [9] and Mühlbauer et al. [12]. The temporal heat source evolution proposed by the former authors is used in conjunction with the respective spatial distribution proposed by the latter authors. Computations with the new model showed an excellent agreement with the experimental data. Furthermore, it has been found that the shape of the entropy wave has a strong influence on the observed maximum pressure fluctuation, even if the maximum temperature fluctuation is kept constant. A shallower shape of the entropy wave induces a lower maximum pressure fluctuation of the indirect noise.

Applying appropriate acoustic boundary conditions at the downstream termination of the EWG is of crucial importance to obtain accurate CFD results $[\underline{9}, \underline{12}]$. So far, characteristic boundary conditions (NSCBCs) have been used to simulate the downstream impedance. In this work, the reported approaches are analyzed with respect to the experimental data. Furthermore, the experimentally measured impedance is incorporated in CFD calculations by means of time-domain impedance boundary conditions (TDIBCs). It has been found that the TDIBC approach leads to a higher accuracy of the CFD data in terms of pressure time series and power spectral densities.

Recent analytical results by Duran et al. [10] propose that the signal measured for the subsonic EWG reference test case is mainly caused by a direct noise mechanism. In this work, the direct noise contribution for the subsonic EWG case is computed numerically. It has been found that the pressure amplitude is approximately 6-7 times lower if only the direct noise is computed. If direct and indirect noise mechanisms are considered, a very good agreement of numerical with experimental data is achieved. Moreover, a timedelay analysis for direct and indirect noise within the EWG test case is presented in this discussion. This analysis also indicates that the peak pressure value is mainly caused by indirect noise. Based on the numerical data and the time-delay analysis shown in this work, it is concluded that the pressure signal measured for the subsonic EWG reference case is mainly generated through an indirect noise mechanism.

\section{Acknowledgments}

The authors gratefully acknowledge F. Bake (DLR, Institute of Propulsion Technology, Berlin) for providing the experimental data and supporting this work. We also want to thank B. Mühlbauer (formerly DLR, Institute of Combustion Technology, Stuttgart) for the fruitful discussions about CFD calculations of the EWG experiment. This work was funded by the German Aerospace Center (DLR) within the project IVTAS.

\section{References}

[1] Leyko, M., Nicoud, F., and Poinsot, T., "Comparison of Direct and Indirect Combustion Noise Mechanisms in a Model Combustor," AIAA Journal, Vol. 47, No. 11, 2009, pp. 2709-2716. doi: $10.2514 / 1.43729$

[2] Smith, M., Aircraft Noise, Vol. 3, Cambridge Aerospace Series, Cambridge Univ. Press, Cambridge, England, U.K., 2004, pp. 57-102.

[3] Strahle, W., "On Combustion Generated Noise," Journal of Fluid Mechanics, Vol. 49, No. 2, 1971, pp. 399-414. doi:10.1017/S0022112071002167

[4] Hassan, H., "Scaling of Combustion-Generated Noise," Journal of Fluid Mechanics, Vol. 66, No. 3, 1974, pp. 445-453. doi:10.1017/S0022112074000292

[5] Howe, M., "Indirect Combustion Noise," Journal of Fluid Mechanics, Vol. 659, 2010, pp. 267-288. doi: $10.1017 / \mathrm{S} 0022112010002466$
[6] Marble, F., and Candel, S., "Acoustic Disturbance from Gas NonUniformities Convected Through a Nozzle," Journal of Sound and Vibration, Vol. 55, No. 2, 1977, pp. 225-243. doi:10.1016/0022-460X(77)90596-X

[7] Bake, F., Richter, C., Mühlbauer, B., Kings, N., Röhle, I., Thiele, F., and Noll, B., "The Entropy Wave Generator (EWG): A Reference Case on Entropy Noise," Journal of Sound and Vibration, Vol. 326, Nos. 3-5, 2009, pp. 574-598. doi:10.1016/j.jsv.2009.05.018

[8] Bake, F., Michel, U., and Roehle, I., "Investigation of Entropy Noise in Aero-Engine Combustors," Journal of Engineering for Gas Turbines and Power, Vol. 129, No. 2, 2007, pp. 370-376. doi: $10.1115 / 1.2364193$

[9] Leyko, M., Moreau, S., Nicoud, F., and Poinsot, T., "Numerical and Analytical Modelling of Entropy Noise in a Supersonic Nozzle with a Shock," Journal of Sound and Vibration, Vol. 330, No. 16, 2011, pp. 3944-3958. doi:10.1016/j.jsv.2011.01.025

[10] Duran, I., Moreau, S., and Poinsot, T., "Analytical and Numerical Study of Combustion Noise Through a Subsonic Nozzle," AIAA Journal, Vol. 51, No. 1, 2013, pp. 42-52. doi:10.2514/1.J051528

[11] Giauque, A., Huet, M., and Clero, F., "Analytical Analysis of Indirect Combustion Noise in Subcritical Nozzles," Journal of Engineering for Gas Turbines and Power, Vol. 134, No. 11, 2012, pp. 1745-1755. doi: $10.1115 / 1.4007318$

[12] Mühlbauer, B., Noll, B., and Aigner, M., "Numerical Investigation of the Fundamental Mechanism for Entropy Noise Generation in AeroEngines," Acta Acustica United with Acustica, Vol. 95, No. 3, 2009, pp. $470-478$. doi:10.3813/AAA.918171

[13] Poinsot, T., and Lele, S., "Boundary Conditions for Direct Simulations of Compressible Viscous Flows," Journal of Computational Physics, Vol. 101, No. 1, 1992, pp. 104-129. doi:10.1016/0021-9991(92)90046-2

[14] Selle, L., Nicoud, F., and Poinsot, T., "Actual Impedance of Nonreflecting Boundary Conditions: Implications for Computation of Resonators," AIAA Journal, Vol. 42, No. 5, 2004, pp. 958-964. doi: $10.2514 / 1.1883$

[15] Widenhorn, A., Noll, B., and Aigner, M., "Impendance Boundary Conditions for the Numerical Simulation of Gas Turbine Combustion Systems," ASME Turbo Expo, Power for Land, Sea and Air, International Gas Turbine Institute, Norcross, GA, Paper GT2008-50445, 2008.

[16] Huber, A., Romann, P., and Polifke, W., "Filter-Based Time-Domain Impedance Boundary Conditions for CFD Applications," ASME Turbo Expo, Power for Land, Sea and Air, International Gas Turbine Institute, Norcross, GA, Paper GT2008-51195, 2008.

[17] Di Domenico, M., Numerical Simulation of Soot Formation in Turbulent Flows, Ph.D. Thesis, Univ. of Stuttgart, Stuttgart, Germany, 2008.

[18] Lourier, J.-M., Di Domenico, M., Noll, B., and Aigner, M., "Implementation of an Efficient Pressure-Based CFD Solver for Accurate Thermoacoustic Computations," 18th AIAA/CEAS Aeroacoustics Conference, AIAA Paper 2012-2089, June 2012.

[19] Kim, J., and Moin, P., "Application of a Fractional-Step Method to Incompressible Navier-Stokes Equations," Journal of Computational Physics, Vol. 59, No. 2, 1985, pp. 308-323. doi:10.1016/0021-9991(85)90148-2

[20] Pierce, C., Progress-Variable Approach for Large-Eddy Simulation of Turbulent Combustion, Ph.D. Thesis, Stanford Univ., Stanford, CA, 2001.

[21] Moureau, V., Bérat, C., and Pitsch, H., "An Efficient Semi-Implicit Compressible Solver for Large-Eddy Simulations," Journal of Computational Physics, Vol. 226, No. 2, 2007, pp. 1256-1270. doi:10.1016/j.jcp.2007.05.035

[22] Widenhorn, A., Noll, B., and Aigner, M., "Accurate Boundary Conditions for the Numerical Simulation of Thermoacoustic Phenomena in Gas-Turbine Combustion Chambers," ASME Turbo Expo, Power for Land, Sea and Air, International Gas Turbine Institute, Norcross, GA, Paper GT2006-90441, 2006, pp. 1-10.

[23] Poinsot, T., and Veynante, D., Theoretical and Numerical Combustion, RT Edwards, Inc., Philadelphia, 2005.

[24] Kreiss, H.-O., "Boundary Conditions for Hyperbolic Differential Equations," Conference on the Numerical Solution of Differential Equations, edited by Watson, G., Vol. 363, Lecture Notes in Mathematics, Springer, Berlin, 1974, pp. 64-74.

[25] Özyörük, Y., Long, L. N., and Jones, M. G., "Time-Domain Numerical Simulation of a Flow-Impedance Tube," Journal of Computational Physics, Vol. 146, No. 1, 1998, pp. 29-57. doi: $10.1006 / \mathrm{jcph} .1998 .5919$ 
[26] Luebbers, R., Hunsberger, F., Kunz, K., Standler, R., and Schneider, M., "A Frequency-Dependent Finite-Difference Time-Domain Formulation for Dispersive Materials," IEEE Transactions on Electromagnetic Compatibility, Vol. 32, No. 3, 1990, pp. 222-227. doi:10.1109/15.57116

[27] Maloney, J., and Smith, G., "The Use of Surface Impedance Concepts in the Finite-Difference Time-Domain Method," IEEE Transactions on Antennas and Propagation, Vol. 40, No. 1, 1992, pp. 38-48. doi: $10.1109 / 8.123351$

[28] Oppenheim, A., and Schafer, R., Discrete-Time Signal Processing, 3rd ed., Person Education Limited, Essex, U.K., 2010, pp. 391-516.

[29] Fung, K., Hongbin, J., and Tallapragada, B., "Impedance and Its TimeDomain Extensions," AIAA Journal, Vol. 38, No. 1, 2000, pp. 30-38. doi: $10.2514 / 2.950$

[30] Fung, K., and Ju, H., "Time-Domain Impedance Boundary Conditions for Computational Acoustics and Aeroacoustics," International Journal of Computational Fluid Dynamics, Vol. 18, No. 6, 2004, pp. 503-511. doi:10.1080/10618560410001673515

[31] Menter, F., "Two-Equation Eddy-Viscosity Turbulence Models for Engineering Applications," AIAA Journal, Vol. 32, No. 8, 1994, pp. $1598-1605$.

doi: $10.2514 / 3.12149$

[32] Dennis, J., and Schnabel, R., Numerical Methods for Unconstrained Optimization and Nonlinear Equations, Vol. 16, Classics in Applied Mathematics, Society for Industrial and Applied Mathematics, Philadelphia, 1987, pp. 221-228.

[33] Levy, E., "Complex Curve Fitting," IRE Transactions on Automatic Control, Vol. 4, No. 1, 1959, pp. 37-43. doi:10.1109/TAC.1959.6429401 